TRANSACTIONS OF THE

AMERICAN MATHEMATICAL SOCIETY

Volume 239, May 1978

\title{
COMPLETE UNIVERSAL LOCALLY FINITE GROUPS
}

BY

\section{KEN HICKIN}

\begin{abstract}
This paper will partly strengthen a recent application of model theory to the construction of sets of pairwise nonembeddable universal locally finite groups [8]. Our result is

THEOREM. There is a set $थ$ of $2^{n_{1}}$ universal locally finite groups of order $\boldsymbol{K}_{1}$ with the following properties:

0.1. If $U \neq V \in \mathcal{Q}$ and $A$ and $B$ are uncountable sugroups of $U$ and $V$, then $A$ and $B$ are not isomorphic.

Let $A$ be an uncountable subgroup of $U \in Q$.

0.2. A does not belong to any proper variety of groups, and

0.3. $A$ is not isomorphic to any of its proper subgroups.

0.4. Every $U \in \mathcal{Q}$ is a complete group (every automorphism of $U$ is inner).
\end{abstract}

Notations. $S<T$ means $S$ is properly contained in $T$;

${ }^{\circ} S=|S|$ is the cardinal (order) of the set $S$;

Aut $G$ and Inn $G$ are respectively the groups of automorphisms and inner automorphisms of the group $G$;

$\operatorname{ISO}(G, H)$ is the set of isomorphisms of $G$ onto $H$;

If $S<G$, then $\operatorname{gp}(S)$ is the smallest subgroup of $G$ containing $S$;

$\omega_{1}$ is the first uncountable ordinal;

An amalgam $A$ is the union of two groups, $A=F \cup H$, where $F$ and $H$ meet in a common subgroup, $F \cap H=E$, and we write

$$
A=\stackrel{F}{E}^{H}
$$

Introduction. A group $U$ is in the class $U L F$ of universal locally finite groups if

$U L F 0 . U$ is locally finite (l.f.),

$U L F 1$. Every finite group is isomorphic to a subgroup of $U$, and

$U L F 2$. If $E$ and $F$ are isomorphic finite subgroups of $U$, then there is some $\varphi \in$ Aut $U$ such that $\varphi(E)=F$.

From these follows

$U L F$ 3. If $A<B$ are finite groups and $\eta: A \rightarrow U$ is an embedding, then

Received by the editors June 8, 1976.

AMS (MOS) subject classifications (1970). Primary 20E25, 20F55, 02H10, 02H15; Secondary 20F20, 20F30, 20B99.

Key words and phrases. Locally finite groups, universal homogeneous groups, complete groups, subgroup-incomparability, regular representation, group amalgams.

- American Mathematical Society 1978 
there is an embedding $g: B \rightarrow U$ such that $g(A)=\eta(A)$. For, by $U L F 1$ there is an embedding $f: B \rightarrow U$; and by $U L F 2$ there is $\alpha \in$ Aut $U$ such that $\alpha f(A)=\eta(A)$. Thus $g=\alpha f$.

Philip Hall introduced the concept of a $U L F$ group in [3] and showed, among other things,

0.5. There is a countable $U L F$ group $C$ which is unique up to isomorphism and

0.6. Every 1.f. group of order $\kappa$ is contained in a $U L F$ group of order $\max \left(\kappa, \aleph_{0}\right)$. In particular, every countable l.f. group is embedded in $C$.

Hall constructed $C$ as a tower of finite symmetric groups with regular embeddings. The regular representation $L<S(L)$, where $S(L)$ is the symmetric group on $L$, has the property

0.7. If $E$ and $F$ are subgroups of $L$ and $\varphi \in \operatorname{ISO}(E, F)$, then for some $x \in S(L), \varphi(a)=x^{-1} a x$ for all $a \in E$.

It follows that $U L F 2$ can be replaced by the more useful

$U L F 4$. If $E$ and $F$ are finite subgroups of $U$ and $\varphi \in \operatorname{ISO}(E, F)$, then for some $x \in U, \varphi(a)=x^{-1} a x$ for all $a \in E$. For, let $G=\operatorname{gp}(E, F)$. By $U L F 3$ let $g: S(G) \rightarrow U$ be an embedding such that $g(G)=G$ where $G<S(G)$ is the regular embedding. By 0.7 there is an $x \in g(S(G))$ as required.

Similarly, $U L F 3$ can be strengthened to

$U L F 5$. If $A \leqslant B$ are finite groups and $\eta: A \rightarrow U$ is an embedding then $\eta$ can be extended to an embedding $g: B \rightarrow U$.

Hall's group $C$ is also obtained in the more general algebraic construction of Jónsson [5] and model theoretic construction of Morley and Vaught [10].

More recently, answering a question of Kegel and Wehrfritz [6], Macintyre and Shelah proved [8]

0.8. For every $\kappa>\aleph_{0}$ there are $2^{\kappa}$ isomorphism types of $U L F$ groups of order $\kappa$ and

0.9 . If $\kappa>\aleph_{0}$ is regular, there are $2^{\kappa}$ pairwise nonembeddable $U L F$ groups of order $k$.

Their proof uses results on unsuperstable first order theories in infinitary logic. In another paper [9] Macintyre gives more information on a question posed in [6]. A l.f. group $H$ is inevitable if $H$ is a subgroup of every $U L F$ group of order $>^{\circ} H$. Hall proved every countable group to be inevitable. Macintyre proves that there is a $U L F$ group of order $\aleph_{1}$ all of whose abelian subgroups are countable. Hence there are no inevitable abelian groups of order $\kappa_{1}$.

The present result strengthens 0.9 for the case $\kappa=\aleph_{1}$. A consequence of 0.1 is that no group of order $\kappa_{1}$ is inevitable. The existence of complete $U L F$ groups contrasts sharply with the fact that Aut $C$ contains a free group on $2^{\mathrm{x}_{0}}$ generators (which will not be proved). 
We will note some basic properties of the class $U L F$.

0.10 . Every countable subset of a $U L F$ group is contained in a subgroup isomorphic to Hall's group $C$.

0.11 . If every finite subset of a group $G$ is contained in a $U L F$ subgroup of $G$, then $G \in U L F$.

The proofs of both use $U L F 4$. For 0.10 the uniqueness of $C$ is also used.

1. The basic construction. This section gives a general procedure for constructing $U L F$ groups of order $\kappa_{1}$, and subsequent sections add specifics to the construction so that the groups obtained satisfy $0.1-0.4$.

The construction requires an amalgamation procedure for finite groups. The following is easily proved using the permutational product of $\mathbf{B}$. $\mathrm{H}$. Neumann [11].

1.1. Lemma. Suppose $E, F$, and $H$ are finite groups, $E<H$, and $g: E \rightarrow F$ is a proper embedding. Then there exists a finite group $G>H$ and an embedding $\varphi: F \rightarrow G$ such that $\varphi g$ is the identity on $E, \varphi F \cap H=E$, and $G=\operatorname{gp}(\varphi F, H)$. Put differently, there is a finite group $G=\operatorname{gp}(A)$ where

$$
A=\underbrace{\varphi F}_{E}
$$

where $\varphi$ is an isomorphism such that $\varphi^{-1} \equiv g$ on $E$.

Outline of the construction. For each ordinal $\alpha<\omega_{1}$ let $C_{\alpha} \cong C$ (Hall's group). We will define proper embeddings $f_{\alpha}: C_{\alpha} \rightarrow C_{\alpha+1}$ obtaining a tower $\cdots \tilde{C}_{\alpha}<\tilde{C}_{\alpha+1} \cdots$ (via $f_{\alpha}$, where $\sim$ is the direct limit map, with continuity at limit $\alpha$ ). By 0.11 each limit group $\tilde{C}_{\alpha} \cong C$, and the union $U$ of the entire tower is a $U L F$ group of order $\aleph_{1}$.

The construction of $f_{\alpha}$. Let $1<F_{1}<\cdots<F_{n} \cdots$ be finite subgroups of $C_{\alpha}$ such that $C_{\alpha}=\cup F_{n}$. We will define a chain of embeddings $\varphi_{n}: F_{n} \rightarrow$ $C_{\alpha+1}$ and put $f_{\alpha}=\bigcup \varphi_{n}$. Let $F_{0}=1$ and $\varphi_{0}: F_{0} \rightarrow C_{\alpha+1}$. Assume $\varphi_{i}$ has been defined for all $i \leqslant n$. Choose a finite subgroup $H_{n}$ of $C_{\alpha+1}$ such that $E_{n}=\varphi_{n}\left(F_{n}\right)<H_{n}$ and $H_{i}<H_{n}$ for all $i<n$ ( $H_{i}$ has been chosen previously). The chain $\left\{H_{n}\right\}$ will be chosen so that $C_{\alpha+1}=\cup H_{n}$. This can be done by arranging that $H_{n}$ contain the $n$th member of some list of $C_{\alpha+1}$.

We now "amalgamate $F_{n+1}$ and $H_{n}$ via $\varphi_{n}$ ", that is, the subgroups $F_{n}$ and $\varphi_{n}\left(F_{n}\right)=E_{n}$ are identified. Specifically, using Lemma 1.1, we obtain

1.2. $G=G_{n}=\mathrm{gp}(A)$ where

$$
A=\underbrace{H_{n+1}}_{E_{n}}
$$


and

1.3. $\varphi \equiv \varphi_{n}$ on $F_{n}$.

By ULF5, $\eta=$ the identity on $H_{n}$ can be extended to an embedding $\tau$ : $G \rightarrow C_{\alpha+1}$. We define $\varphi_{n+1}=\tau \varphi: F_{n+1} \rightarrow C_{\alpha+1}$ and observe

1.4. $\varphi_{n+1} \equiv \varphi_{n}$ on $F_{n}$, which follows from 1.3 and the fact that $\tau \equiv 1$ on $H_{n}>E_{n}$.

Thus there is a unique embedding $f_{\alpha}: C_{\alpha} \rightarrow C_{\alpha+1}$ such that $f_{\alpha} \equiv \varphi_{n}$ on $F_{n}$ for all $n>0$.

We will list the main properties of $f_{\alpha}$ for later use.

1.5. Summary of notation. $C_{\alpha}=\bigcup F_{n}, C_{\alpha+1}=\cup H_{n}, f_{\alpha}: C_{\alpha} \rightarrow C_{\alpha+1}, f_{\alpha} \equiv$ $\varphi_{n}$ on $F_{n}, E_{n}=\varphi_{n}\left(F_{n}\right)=f_{\alpha}\left(F_{n}\right)<H_{n}$ and so $f_{\alpha}\left(C_{\alpha}\right)=\bigcup E_{n}<C_{\alpha+1}$.

1.6. $H_{n} \cap E_{n+1}=E_{n}$ for all $n \geqslant 0$.

Proof. Since $\tau \equiv 1$ on $H_{n}$, from 1.2 we have

$$
\tau(A)=\underbrace{E_{n+1}}_{E_{n}}{ }^{H_{n}}
$$

where $E_{n+1}=\tau \varphi\left(F_{n+1}\right)=\varphi_{n+1}\left(F_{n+1}\right)$.

1.7. $f_{\alpha}\left(C_{\alpha}\right) \cap H_{m}=E_{m}$ for all $m \geqslant 0$. In particular, $f_{\alpha}$ is a proper embedding.

Proof. Assume inductively $E_{n} \cap H_{m}=E_{m}$ for some $n \geqslant m, n=m$ being trivial. Then $E_{n+1} \cap H_{m}=E_{n+1} \cap H_{n} \cap H_{m}=E_{n} \cap H_{m}=E_{m}$ (using 1.6). Since $f_{\alpha}\left(C_{\alpha}\right)=\cup E_{n}, 1.7$ follows.

1.8. Amalgamating property of $f_{\alpha}$. Let $J_{n}=\operatorname{gp}\left(E_{n+1}, H_{n}\right)<C_{\alpha+1}$ and let $G=G_{n}=\operatorname{gp}(A)$, where

$$
A=\underbrace{H_{n}}_{E_{n}}
$$

and $\varphi \equiv f_{\alpha}$ on $F_{n}$, be obtained from Lemma 1.1. There is some $\tau \in$ $\operatorname{ISO}\left(G_{n}, J_{n}\right)$ such that $\tau \equiv 1$ on $H_{n}$ and $\tau \varphi \equiv f_{\alpha}$ on $F_{n+1}$. (The embedding $\tau$ defined above has these properties.)

These embeddings are represented in the following diagram in which slanted or vertical lines are inclusions:

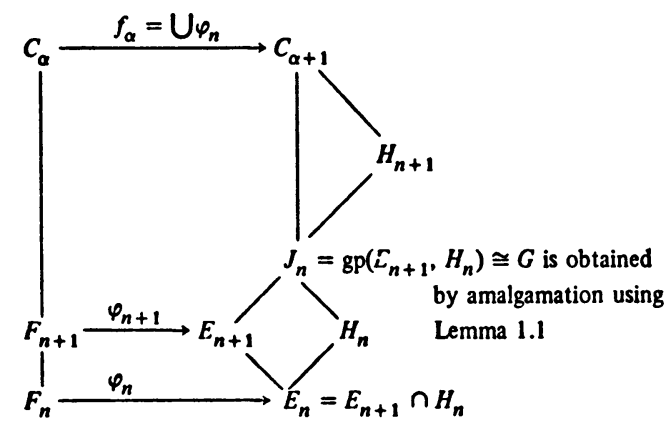


1.9. Summary of the construction of $U$. A $U L F$ group $U$ of power $\kappa_{1}$ is obtained as the direct union of the groups $C_{\alpha} \cong C$ via the embeddings $f_{\alpha}$ : $C_{\alpha} \rightarrow C_{\alpha+1}$ for each $\alpha<\omega_{1}$ by imposing continuity at each limit ordinal.

Specifically, there exists a direct limit map $\sim: \cup\left\{C_{\alpha}\right\} \rightarrow U$ with the following properties. Let $U_{\alpha}=\tilde{C}_{\alpha}$.

1.10. : $C_{\alpha} \rightarrow U_{\alpha}$ is an isomorphism and $U=\bigcup\left\{U_{\alpha}: \alpha<\omega_{1}\right\}$.

1.11. For all $x \in C_{\alpha}, \tilde{x}=\widetilde{f_{\alpha}(x)}$, and so $U_{\alpha}<U_{\alpha+1}$.

1.12 (continuity). If $\lambda>0$ is a limit ordinal, then $U_{\lambda}=\bigcup\left\{U_{\beta}: \beta<\lambda\right\}\left({ }^{\mathrm{l}}\right)$.

From 1.11 it follows that for all $\alpha<\omega_{1}$

1.13. $\tilde{F}_{n}=\tilde{E}_{n}$ (see 1.5), and

1.14. The embeddings $f_{\alpha}\left(C_{\alpha}\right)<C_{\alpha+1}$ and $U_{\alpha}<U_{\alpha+1}$ are isomorphic.

Each embedding $f_{\alpha}$ is determined by the choices made for (1) the chains $\left\{H_{n}^{\alpha}\right\}=\left\{H_{n}\right\}$ and $\left\{F_{n}^{\alpha}\right\}=\left\{F_{n}\right\}$ of finite subgroups of $C_{\alpha+1}=U \cdot H_{n}^{\alpha}$ and $C_{\alpha}=\cup F_{n}^{\alpha}$, (2) the amalgamating groups $G=G_{n}$ supplied by Lemma 1.1 as described in 1.8, and (3) the embeddings $\tau: G_{n} \rightarrow C_{\alpha+1}$. The choices made for $\tau$ and for the chains $\left\{H_{n}^{\alpha}\right\}$ will be immaterial to the results of the paper.

We will see that the amalgamating group $G$ can be chosen so that certain subgroups of $G$ have a prescribed numerical structure, namely that any given prime dominates their order. It will follow that if $x \in U_{\alpha+1}-U_{\alpha}$, then $\mathrm{gp}(x, y)$ has this structure for almost all $y \in U_{\alpha}$; and this will give control over the numerical structure of all uncountable subgroups of $U$. This will be done in $\$ 2$ where $0.1-0.3$ will be proved. In $\$ 3$ the effect of the chains $\left\{F_{n}^{\alpha}\right\}$ on the structure of $U$ will be considered, and this will lead to the proof of 0.4 .

\section{Subgroup-incomparable $U L F$ groups.}

Definition. The prime $p$ dominates the finite group $G$ if ${ }^{\circ} P>$ index of $P$ in $G$, where $P$ is a $p$ Sylow subgroup of $G$.

The next lemma prescribes the choice of the amalgamating groups $G$ obtained from Lemma 1.1.

2.1. LemMa. Let $p$ be a prime, $r \geqslant 1$, and $w_{1}, \ldots, w_{d}$ nontrivial words in a countable free group. There is a group $G$ meeting the conditions in Lemma 1.1 such that, for all $x \in H-E$ and $y \in \varphi F-E$, (i) $p$ dominates $\mathrm{gp}(x, y)$, (ii) ${ }^{\circ} \mathrm{gp}(x, y)>r$ and (iii) if $x^{2} \notin E$, then $w_{i} \not 1$ in $\mathrm{gp}(x, y), 1<i<d$.

PROof. Let $Q$ be a finite group with the properties of $G$ in Lemma 1.1, so $Q=\operatorname{gp}(\varphi F, H)$, etc. Let $Q=\varphi F{ }_{E} H$ (the amalgamated free product) and let $Q \cong \mathscr{Q} / K$ (naturally). $K$ is a free group [7, p. 228, (5) and (6)] and is finitely generated since $\mathscr{Q}$ is f.g. and $\mathscr{Q} / K$ is finite [13, 8.4.13]. Using a well-known property of free groups, for every finite $S<K-1$ there exists $P<K$ such that $P$ is a characteristic subgroup of $\mathcal{Q}, S<K-P$, and $K / P$

( ) Note that $\sim$ is constructed inductively. $U_{\lambda} \simeq C$ by 0.11 and the uniqueness of $C$; and $\sim$ : $C_{\lambda} \rightarrow U_{\lambda}$ is an arbitrary isomorphism. 
is a finite $p$ group. The main results needed to prove this are $[13,7.1 .6$ and 8.4.16] and [1, Theorem 2.2].

Let $x \in H-E$ and $y \in \varphi F-E$. If $x^{2} \notin E$, then $x y x$ and $y x$ generate a free subgroup of $\mathscr{Q}[12$, p. 510]. Hence,

2.2. If $x^{2} \notin E$, then $K \cap \mathrm{gp}(x, y)=K_{x y}$ is a free group of rank $>1$.

We can choose a characteristic subgroup $P=P(x, y)$ of $\mathcal{Q}$ so that $P<K$, $K / P$ is a finite $p$ group, and, defining $\Phi=P K_{x y} / P \cong K_{x y} / P \cap \operatorname{gp}(x, y)$,

2.3. ${ }^{\circ} \Phi>\max \left({ }^{\circ} Q, r\right)$ and if $x^{2} \notin E$, then $w_{i} \neq 1$ in $\Phi, 1<i<d$. (The first condition is met if $X \cup\left(X X^{-1}-1\right)<K-P$ where $X\left\langle K_{x y}-1{ }^{o} X\right\rangle$ $\max \left({ }^{\circ} Q, r\right)$, while the second is met using 2.2 if $K_{x y}-P$ contains words in $K_{x y}$ of structure similar to each $w_{i}$.)

Define $N=\cap\{P(x, y): x \in H-E, y \in \varphi F-E\}$ and $G=\mathbb{Q} / N$ and let $\beta: \mathbb{Q} \rightarrow G$ be the factor map. $G$ is finite because $N$ is obtained as a finite intersection of subgroups of finite index. Let $\Omega=N K_{x y} / N<\beta \operatorname{gp}(x, y) . \Omega$ is a $p$ group because $K / N$ is. Since $\Phi$ is a factor of $\Omega,{ }^{\circ} \Omega>\max \left({ }^{\circ} Q, r\right)$, and it follows that

2.4. $p$ dominates $\beta \mathrm{gp}(x, y)$, because $\beta \mathrm{gp}(x, y) / \Omega \cong \mathrm{gp}(x, y) / K_{x y}$ and so has order $<^{\circ} Q$, and

2.5. $\beta \mathrm{gp}(x, y)>r$ and if $x^{2} \notin E$, then $w_{i} \neq 1$ in $\beta \mathrm{gp}(x, y), 1<i<d$, (by 2.3 because $\Phi$ is a factor of $\Omega$ ).

If we identify $H$ with $\beta H<G$ and take $\beta \varphi: F \rightarrow G$ as the new $\varphi$, then the conclusions of our lemma follow from 2.4 and 2.5 .

2.6. Definition of $\Pi\left(f_{\alpha}\right)$. Let $\Pi$ be an infinite set of primes and let $f_{\alpha}$ : $C_{\alpha} \rightarrow C_{\alpha+1}$ be the embedding of 1.9. The property $\Pi\left(f_{\alpha}\right)$ holds if (refer to 1.5 ) for all $n \geqslant 0, x \in H_{n}-E_{n}$, and $y \in E_{n+1}-E_{n}$, (l) $p_{n}$ domaintes $\mathrm{gp}(x, y)$ where $\left(p_{i}\right)$ is some list of $\Pi$, (2) ${ }^{\circ} \mathrm{gp}(y, x)>\left|E_{n+1}\right|^{2}$, and (3) if $x^{2} \notin E$, then $w_{n} \neq 1$ in $\operatorname{gp}(y, x), 1<i \leqslant n$, where $\left(w_{i}\right)$ is some list of the nontrivial words in a countable free group.

2.7. LemMA. The groups $G_{n}$ of 1.8 can be chosen so that $\Pi\left(f_{\alpha}\right)$ holds.

PRoof. $G_{n}=\mathrm{gp}(A)$, where

$$
A=\underbrace{\varphi F_{n+1}}_{E_{n}} H_{n}
$$

is obtained from Lemma 1.1. Lemma 2.1 allows us to choose $G_{n}$ so that for all $x \in H_{n}-E_{n}$ and $y \in \varphi F_{n+1}-E_{n}, p_{n}$ dominates $\operatorname{gp}(y, x),{ }^{\circ} \mathrm{gp}(y, x)>$ $\left|E_{n+1}\right|^{2}=r$, and if $x^{2} \notin E_{n}$, then $w_{i} \not 1$ in $\mathrm{gp}(y, x), 1<i<n$. Now the conclusions (1)-(3) follow by applying $\tau: G_{n} \rightarrow \mathrm{gp}\left(E_{n+1}, H_{n}\right)$.

2.8. Lemma. Assume $\Pi\left(f_{\alpha}\right)$ holds. Let $x \in C_{\alpha+1}-f_{\alpha}\left(C_{\alpha}\right)$ and let $Y<$ $f_{\alpha}\left(C_{\alpha}\right)$ be infinite. Then, 
(1) $\{p: p$ dominates $\operatorname{gp}(y, x)$ for some $y \in Y\}=\Lambda \cup L$ where $\Lambda \leqslant \Pi$ is infinite and $L$ is finite,

(2) $\mathrm{gp}(Y, x)$ does not belong to a proper variety, and

(3) Define $Y_{i}=\left\{y \in Y: p_{i}\right.$ dominates $\left.\operatorname{gp}(y, x)\right\}$. There is some $n \geqslant 0$ such that, for all $i \geqslant n, Y_{i}=Y \cap\left(E_{i+1}-E_{i}\right)$ and hence, for all $j>n$, we have

2.9. ${ }^{o} Y \cap E_{j}={ }^{o} Y \cap E_{n}+\sum_{i=n}^{j-1} Y_{i}$.

Proof. Refer to 1.5 and 1.8. Let $z \in C_{\alpha+1}-f_{\alpha}\left(C_{\alpha}\right)$. For some $m=m(z), z$ $\in H_{m}$. Let $y \in Y \cap\left(E_{j+1}-E_{j}\right)$ for some $j \geqslant m$. Since $z \in H_{j}-E_{j}$ we have by $\Pi\left(f_{\alpha}\right)$

2.10. $p_{j}$ dominates $\operatorname{gp}(y, z),{ }^{o} \mathrm{gp}(y, z)>\left|E_{j+1}\right|^{2}$, and if $z^{2} \notin E_{j}$, then $w_{i} \neq 1$ in $\operatorname{gp}(y, z), 1 \leqslant i \leqslant j$. Recall that $p_{j} \in \Pi$.

To prove (1), define $\Lambda=\{p: p$ dominates $\operatorname{gp}(y, x)$ for some $y \in Y-$ $\left.E_{m(x)}\right\}$. Taking $z=x$ in 2.10 , we have $\Lambda \leqslant \Pi$. Since the groups $E_{i}$ are finite, $y$ can be chosen so that $j$ is arbitrarily large. Hence $\Lambda$ is infinite and (1) follows.

To prove (2), we first choose $y_{0} \in Y-E_{m(x)}$ such that $y_{0} \in E_{n+1}-E_{n}$ where $p_{n} \neq 2$. Taking $z=x$ and $j=n$ in 2.10 , we have ${ }^{\circ} \mathrm{gp}\left(y_{0}, x\right)>\left|E_{n+1}\right|^{2}$ and $p_{n}$ dominates $\mathrm{gp}\left(y_{0}, x\right)$. It follows that no $p_{n}$ Sylow subgroup of $\operatorname{gp}\left(y_{0}, x\right)$ is contained in $E_{n+1}$. So, there is a $p_{n}$-element $z \in \operatorname{gp}(x, y)-E_{n+1}$. Note that $z \in \operatorname{gp}\left(E_{n+1}, H_{n}\right) \leqslant H_{n+1}$ and thus $z, z^{2} \notin \cup E_{j}=f_{\alpha}\left(C_{\alpha}\right)$ because of 1.7. Now we can apply 2.10: For arbitrarily large values of $j \geqslant m(z)=n+1$, there is some $y \in Y \cap\left(E_{j+1}-E_{j}\right)$, and $w_{i} \neq 1$ in $\operatorname{gp}(y, z)$ for all $i \leqslant j$. Hence $\operatorname{gp}(Y, z) \leqslant \operatorname{gp}(Y, x)$ belongs to no proper variety.

To prove (3), let $x \in H_{m}$ and define $P=\{p: p$ dominates $\operatorname{gp}(y, x)$ for some $\left.y \in Y \cap E_{m}\right\}$. Since $P$ is finite we can choose $n \geqslant m$ so that, for all $i \geqslant n, p_{i} \notin P$. Suppose $i \geqslant n$ and $y \in Y_{i}$. We have $y \notin E_{m}$ because $p_{i} \notin P$, and so $y \in E_{r+1}-E_{r}$ for some $r \geqslant m$. Since $x \in H_{r}, p_{r}$ dominates $\operatorname{gp}(y, x)$ by $\Pi\left(f_{\alpha}\right)$. Thus $r=i$ and we conclude $Y_{i}=Y \cap\left(E_{i+1}-E_{i}\right)$. The equality 2.9 is immediate because $Y \cap E_{j}$ is the disjoint union of the sets involved.

2.11. THEOREM. There is a set $\mathscr{U}$ of $2^{\aleph_{1}}$ ULF groups of order $\aleph_{1}$ satisfying 0.1-0.3.

Proof of 0.1 AND 0.2. By a well-known set theoretic lemma [14, p. 451] there is a set $\mathscr{P}$ such that each $\Pi \in \mathscr{P}$ is an infinite set of primes, ${ }^{\circ} \mathscr{P}=\aleph_{1}$, and

2.12. If $\Pi \neq \Gamma \in \mathcal{P}$, then $\Pi \cap \Gamma$ is finite.

Now apply the lemma again to obtain a set 2 such that, for each $\mathfrak{V} \in \mathcal{Q}$, $\mathfrak{V}<\mathscr{P}, o \mathfrak{V}=\kappa_{1},{ }^{\circ} \mathcal{Q}=2^{\kappa_{1}}$, and

2.13. If $\mathscr{V} \neq \mathcal{T} \in 2$, then ${ }^{\circ} \mathcal{V} \cap \mathcal{T} \leqslant \kappa_{0}$.

Let $\mathfrak{V}_{0} \in \mathcal{2}$. Since ${ }^{o \mathcal{V}_{0}}=\kappa_{1}$, let $\mathfrak{V}_{0}=\left\{\Pi_{\alpha}: \alpha<\omega_{1}\right\}$. We now construct a $U L F$ group $U=U_{0}$ as in 1.9 with the additional condition that $\Pi_{\alpha}\left(f_{\alpha}\right)$ holds for all $\alpha<\omega_{1}$. This is possible by Lemma 2.7 . 
Define $थ=\left\{U_{0}: \mho_{0} \in \mathcal{Q}\right\}$.

Let $U_{0}=U \in \mathcal{Q}$. Recall (see 1.9) that $U=U\left\{U_{\alpha}: \alpha<\omega_{1}\right\}, U_{\alpha} \approx C$, so every countable subset of $U$ is contained in some $U_{\alpha}$. Let $T$ be an uncountable subgroup of $U$ and put $T_{\alpha}=T \cap U_{\alpha}$.

Let $Y<T,{ }^{\circ} Y=\aleph_{0}$. For each $x \in U$ define $P(Y, x)=\{p$ : $p$ dominates $\operatorname{gp}(y, x)$ for some $y \in Y\}$. Consider the following property of a pair $(B, \mathcal{V})$, $B<U, \mathcal{T} \in 2$ :

2.14. $Y<B<T,{ }^{\circ} B=\aleph_{0}$, and for all $x \in T-B$ there is some $\Pi \in \mathcal{V}$ such that $P(Y, x) \cap \Pi$ is infinite.

We will prove two propositions:

2.15. If $B<U=U_{0}$ and $\mathcal{V} \in 2$ satisfy 2.14 , then $\mathcal{V}=\mho_{0}$.

2.16. Let $\beta$ be such that $Y<T_{\beta}$. Then 2.14 is satisfied if $B=T_{\beta}$ and $\mathfrak{V}=\mathfrak{V}_{0}$

PROOF OF 2.16. Let $x \in T-T_{\beta}$. Then, by continuity (1.12), $x \in T_{\alpha+1}-$ $T_{\alpha}$ for some $\alpha>\beta$. Note $Y \leqslant T_{\alpha}$. Since the embeddings $U_{\alpha}<U_{\alpha+1}$ and $f_{\alpha}\left(C_{\alpha}\right)<C_{\alpha+1}$ are isomorphic (1.14) and $\Pi_{\alpha}\left(f_{\alpha}\right)$ holds, parts (1) and (2) of Lemma 2.8 imply

2.17. $P(Y, x)=\Lambda \cup L$ where $\Lambda<\Pi_{\alpha}$ is infinite and $L$ is finite, and

2.18. $\mathrm{gp}(Y, x)$ does not belong to any proper variety.

Thus $P(Y, x) \cap \Pi_{\alpha}$ is infinite and 2.16 follows since $\Pi_{\alpha} \in \mathcal{V}_{0}$.

PROOF OF 2.15. Notice that, because of 2.12, 2.17 implies

2.19. If $x \in T-T_{\beta}$, then $\Pi_{\alpha}$ is the unique $\Pi \in \mathcal{P}$ such that $P(Y, x) \cap \Pi$ is infinite where $x \in T_{\alpha+1}-T_{\alpha}$.

Now suppose $B<U_{0}$ and $\checkmark \in \mathcal{Q}$ satisfy 2.14. Let $Y<T_{\beta}$ and let $x \in T-\left(B \cup T_{\beta}\right)$. By 2.19 we have

2.20. $\Pi_{\alpha} \in \mathcal{V}_{0} \cap \mathcal{V}$ where $x \in T_{\alpha+1}-T_{\alpha}$.

Since ${ }^{\circ} T=\kappa_{1}, 2.20$ must hold for an unbounded set of $\alpha<\omega_{1}$, which implies $\circ \mathfrak{V}_{0} \cap \mathcal{V}=\kappa_{1}$, and from 2.13 we now conclude $\mathfrak{V}_{0}=\mathfrak{V}$ as desired.

It follows that $\Upsilon_{0}$ is determined uniquely by the condition 2.14 from the structure of any uncountable subgroup $T$ of $U_{0}$, and this proves 0.1 . Note that 0.2 is immediate from 2.18 because $\mathrm{gp}(Y, x)<T$.

Proof of 0.3. Let $U \in \mathcal{Q}$ and let $S<T$ be uncountable subgroups of $U$. Suppose there exists $\sigma \in \operatorname{ISO}(S, T)$. Let $S_{\alpha}=U_{\alpha} \cap S$. By the continuity of the chain $\left\{U_{\alpha}\right\}$, there is some $\beta$ such that $\sigma S_{\beta}=T_{\beta}$ and $T_{\beta}$ is infinite. Let $\alpha>\beta$. By 2.19 , letting $Y=T_{\beta}$, we have $T_{\alpha+1}-T_{\alpha}=\left\{x \in T-T_{\beta}\right.$ : $P\left(T_{\beta}, x\right) \cap \Pi_{\alpha}$ is infinite $\}$, and, replacing $T$ by $S$, we likewise have $S_{\alpha+1}-$ $S_{\alpha}=\left\{x \in S-S_{\beta}: P\left(S_{\beta}, x\right) \cap \Pi_{\alpha}\right.$ is infinite $\}$. Since $\sigma S_{\beta}=T_{\beta}$ it follows that $\sigma\left(S_{\alpha+1}-S_{\alpha}\right)=T_{\alpha+1}-T_{\alpha}$, and

2.21. $\sigma S_{\alpha}=T_{\alpha}$ for all $\alpha \geqslant \beta$.

Suppose $\alpha>\beta$ and there exists $z \in S_{\alpha+1}-S_{\alpha}$. Let $x=\sigma(z) \in T_{\alpha+1}-$ $T_{\alpha}$. Recall that $U_{\alpha}=\cup \tilde{E}_{n}$ (see 1.9). Let $Y=T_{\alpha}$ in Lemma 2.8; part (3) 
implies that $n=n_{1}$ exists such that, for all $j>n,{ }^{\circ} T_{\alpha} \cap \tilde{E}_{j}={ }^{o} T_{\alpha} \cap \tilde{E}_{n}+$ $\sum_{i=n}^{j-1} Y_{i}$ where $Y_{i}=\left\{y \in T_{\alpha}: p_{i}\right.$ dominates $\left.\mathrm{gp}(y, x)\right\}$. Putting $Y=S_{\alpha}$ gives the existence of $n=n_{2}$ such that, for all $j>n,{ }^{o} S_{\alpha} \cap \tilde{E}_{j}={ }^{o} S_{\alpha} \cap \tilde{E}_{n}+$ $\sum_{i=n}^{j-10} Y_{i}^{\prime}$ where $Y_{i}^{\prime}=\left\{y \in S_{\alpha}: p_{i}\right.$ dominates $\left.\operatorname{gp}(y, z)\right\}$. Since $\sigma S_{\alpha}=T_{\alpha}$ and $\sigma(z)=x$, we have $\sigma Y_{i}=Y_{i}^{\prime}$, and so ${ }^{\circ} Y_{i}={ }^{o} Y_{i}^{\prime}$, for all $i$. Hence, if $n=$ $\max \left(n_{1}, n_{2}\right)$ we conclude

2.22. $\left|{ }^{\circ} T_{\alpha} \cap \tilde{E}_{j}-{ }^{o} S_{\alpha} \cap \tilde{E}_{j}\right|<^{\circ} E_{n}$ for all $j>n$.

(The fact that $n$ can be increased in 2.9 follows from the full statement of (3).) Assume $S_{\alpha}<T_{\alpha}$. Since $S_{\alpha}=\bigcup\left\{S_{\alpha} \cap \tilde{E}_{j}\right\}$ is infinite, there is some $j>n$ such that ${ }^{\circ} S_{\alpha} \cap \tilde{E}_{j}>{ }^{o} E_{n}$ and $S_{\alpha} \cap \tilde{E}_{j}<T_{\alpha} \cap \tilde{E}_{j}$, and this contradicts 2.22 . Hence $S_{\alpha}=T_{\alpha}$ for an unbounded set of $\alpha<\omega_{1}$, and so $S=T$.

3. Complete ULF groups. The next lemma shows that the numerical structure of the groups $U$ constructed in Theorem 2.11 places a severe restriction on their automorphisms.

3.1. Lemma. Let $थ$ be the set of groups constructed in Theorem 2.11. Let $U \in \mathcal{Q}$ and $\theta \in$ Aut $U$. (a) Let $\tau<\omega_{1}$ be minimal such that $\theta U_{\tau}=U_{\tau}$. Then, for all $\alpha \geqslant \tau, \theta U_{\alpha}=U_{\alpha}$; (b) Suppose $\alpha \geqslant \lambda \geqslant \tau$. Using the notation of 1.5 and 1.9, we have $U_{\alpha}=\cup \tilde{E}_{n}$ and $U_{a+1}=\cup \tilde{H}_{n}$. Let $D_{n}^{\alpha}=D_{n}=U_{\lambda} \cap \tilde{E}_{n}$. Then $\theta D_{n}=D_{n}$ for all but finitely many values of $n$. In particular, taking $\lambda=\alpha$, $\theta \tilde{E}_{n}=\tilde{E}_{n}$ for almost all $n$.

Proof of (a). This is actually a special case of 2.21 , letting $S=T=U$, $\beta=\tau$, and $\sigma=\theta$.

Proof of (b). Choose any $x \in U_{\alpha+1}-U_{\alpha}$. Then $\theta(x) \in U_{\alpha+1}-U_{\alpha}$ by part (a). Choose $m$ so that $x, \theta(x) \in \tilde{H}_{m}$. Suppose $\theta D_{n} \neq D_{n}$ for infinitely many $n$. Then the set $\left\{a \in U_{\alpha}: a \in D_{j+1}-D_{j}\right.$ and $\left.\theta(a) \notin D_{j+1}\right\}$ is infinite, and we can choose $j \geqslant m$ and $a \in D_{j+1}-D_{j}$ so that $\theta(a) \notin D_{j+1}$. By part (a), $\theta(a) \in D_{n+1}-D_{n}$ for some $n>j$. Since $\Pi_{\alpha}\left(f_{\alpha}\right)$ holds we have (1) $p_{j}$ dominates $\operatorname{gp}(a, x)$ because $a \in \tilde{E}_{j+1}-\tilde{E}_{j}$ and $x \in \tilde{H}_{j}-\tilde{E}_{j}$, and similarly (2) $p_{n}$ dominates $\operatorname{gp}(\theta(a), \theta(x))$ where $p_{j}$ and $p_{n}$ are the $j$ th and $n$th members of a list of $\Pi_{\alpha}$. Since $\theta \in$ Aut $U_{\alpha+1}$ we must have $p_{j}=p_{n}$, and this contradiction proves (b).

The next lemma gives a convenient criterion for the completeness of a group $U$.

3.2. Lemma. Refer to 1.10. Suppose $\theta \in$ Aut $U$. Let $\theta_{\alpha}=$ the restriction of $\theta$ to $U_{\alpha}$. Suppose that for all limit ordinals $\lambda<\omega_{1}$ such that $\theta U_{\lambda}=U_{\lambda}$ we have $\theta_{\lambda} \in \operatorname{Inn} U_{\lambda}$. Then $\theta \in \operatorname{Inn} U$.

The proof uses the well-known regressive function principle for $\omega_{1}$. Continuity of the chain $\left\{U_{\alpha}\right\}$ (1.12) guarantees that $\theta U_{\lambda}=U_{\lambda}$ for all $\lambda$ belonging to a closed, unbounded subset of $\omega_{1}$. For each such $\lambda$, choose 
$x_{\lambda} \in U_{\lambda}$ such that $\theta_{\lambda}=$ conjugation by $x_{\lambda}$. According to the regressive function principle, the function $\lambda \rightarrow x_{\lambda}$ is constant on some unbounded subset $S$ of $\omega_{1}$, that is, $x_{\lambda}=x$ for all $\lambda \in S$. So $\theta=$ conjugation by $x$. More details can be found in [4, Lemma 3.3 and the proof of Theorem 6.5] or, more appropriately, [2].

Lemmas 3.1 and 3.2 provide a method to obtain complete groups $U \in \mathcal{Q}$ : For each limit group $U_{\lambda}, \lambda \geqslant \tau$, we would like the conditions in 3.1(b), namely that $\theta D_{n}^{\alpha}=D_{n}^{\alpha}$ for all $\alpha \geqslant \lambda$ and for almost all $n$ to guarantee that $\theta_{\lambda} \in \operatorname{Inn} U_{\lambda}$. Since $D_{n}^{\alpha}=U_{\lambda} \cap \tilde{F}_{n}^{\alpha}$ (see 1.5 and 1.13), the chains $\left\{D_{n}^{\alpha}\right\}$ depend on the chains $\left\{F_{n}^{\alpha}\right\}, \alpha \geqslant \lambda$, which we are free to choose as we please (see 1.9). It is not yet clear exactly how many chains $\left\{D_{n}^{\alpha}\right\}$ (for various $\alpha \geqslant \lambda)$ must be utilized to smother the outer automorphisms of $U_{\lambda}$. There might in fact be a single chain $\left\{D_{n}^{\lambda}\right\}=\left\{\tilde{F}_{n}^{\lambda}\right\}$ which suffices by itself: that is, if $\theta \tilde{F}_{n}^{\lambda}=\tilde{F}_{n}^{\lambda}$ for almost all $n$, then $\theta_{\lambda} \in \operatorname{Inn} U_{\lambda}$. However, the present proof will use two chains $\left\{\tilde{F}_{n}^{\lambda}\right\}$ and $\left\{D_{n}^{\lambda+1}\right\}=\left\{U_{\lambda} \cap \tilde{F}_{n}^{\lambda+1}\right\}=\left\{\tilde{I}_{n}^{\lambda}\right\}$. These chains will now be defined.

3.3. Definition of the chains $\left\{F_{i}^{\lambda}\right\}$ AND $\left\{I_{i}^{\lambda}\right\}$ of $C_{\lambda}$. Recalling Hall's construction of $C$ (see Introduction), let $C_{\lambda}=\cup L_{n}$ where $L_{1}$ is a symmetric group of degree $3, L_{n+1} \cong$ the symmetric group on $L_{n}$ and $L_{n}<L_{n+1}$ is the left regular embedding. Let $R_{n}=$ the centralizer of $L_{n}$ in $L_{n+1}$. It is easily shown that $R_{n}$ is the right regular representation of $L_{n}$ in $L_{n+1}$. So $\mathrm{gp}\left(L_{n}, R_{n}\right)$ $=L_{n} \oplus R_{n}<L_{n+1}$. Since $R_{n}$ is a symmetric group of degree $d \geqslant 3$, there is a chain $R_{n}^{1}<\cdots<R_{n}^{d-2}=R_{n}$ where $R_{n}^{i}$ is the subgroup fixing each of $d-(i+2)$ permuted objects. Note $R_{n}^{1} \cong L_{1}$ and let $A_{n} \neq B_{n}<R_{n}^{1}$ be subgroups of order 2 . The chain $\left\{F_{i}^{\lambda}\right\}$ will consist of all the members of segments of the form $\cdots L_{n}<L_{n} \oplus A_{n}<L_{n} \oplus R_{n}^{1}<\cdots<L_{n} \oplus R_{n}^{d-2}$ $<L_{n+1} \cdots(n \geqslant 1)$. The chain $\left\{I_{i}^{\lambda}\right\}$ is defined identically, except we replace each $A_{n}$ by $B_{n}$ in the second member of each segment. Note that $\left\{L_{n}\right\}$ is a subchain of both $\left\{F_{n}^{\lambda}\right\}$ and $\left\{I_{n}^{\lambda}\right\}$.

3.4. Lemana. If $\Gamma \in$ Aut $C_{\lambda}$ and almost all the members of the chains $\left\{F_{i}^{\lambda}\right\}=\left\{F_{i}\right\}$ and $\left\{I_{i}^{\lambda}\right\}=\left\{I_{i}\right\}$ are invariant under $\Gamma$, then $\Gamma \in \operatorname{Inn} C_{\lambda}$.

Proof. The proof depends on the completeness of the finite symmetric group [13, 11.4]. Let $m$ be such that for all $i \geqslant m, F_{i}$ and $I_{i}$ are invariant under $\Gamma$. Choose $n$ so that $F_{m} \leqslant L_{n}$. Thus $\Gamma L_{n}=L_{n}$ and since $L_{n}$ is a complete group, there is some $x \in L_{n}$ such that

3.5. $\Gamma(a)=x^{-1} a x$ for all $a \in L_{n}$.

We will show by induction that 3.5 is valid for all $a \in C_{\lambda}$. Assume that 3.5 holds for all $a \in L_{j}, j \geqslant n$. Since $\Gamma L_{j+1}=L_{j+1}$ and $L_{j+1}$ is complete, for some $y \in L_{j+1}, \Gamma(a)=y^{-1} a y$ for all $a \in L_{j+1}$. Hence $z=y x^{-1}$ centralizes $L_{j}$, implying $z \in R_{j}$. All of the groups in the series $L_{j}<L_{j} \oplus A_{j}$ or $L_{j} \oplus B_{j}<$ 
$L_{j} \oplus R_{j}^{1}<\cdots<L_{j} \oplus R_{j}^{d-2}$ are invariant under $\Gamma$ and hence under conjugation by $z$, and it follows (e.g. by Krull-Schmidt) that each of the groups $A_{j}, B_{j}, R_{j}{ }^{1}, \ldots, R_{j}^{d-2}=R_{j}$ are also $z$-invariant. However, from the definitions of these subgroups (see 3.3), it is seen that only the identity of $R_{j}$ normalizes all of them. Consequently, $z=1, x=y$, and the induction is complete.

We can now give the condition for the completeness of $U$.

3.6. Lemma. Let $U \in \mathcal{Q}=$ the set of groups constructed in Theorem 2.11 . Suppose that for all limit $\lambda<\omega_{1}$ the chain $\left\{F_{i}^{\lambda}\right\}$ is defined as in 3.3 and the chain $\left\{F_{i}^{\lambda+1}\right\}$ is chosen so that

3.7. $\tilde{I}_{i}^{\lambda}=U_{\lambda} \cap \tilde{F}_{i}^{\lambda+1}\left(=D_{i}^{\lambda+1}\right)$ for all $i \geqslant 0$.

Then Aut $U=\operatorname{Inn} U$.

Proof. Let $\theta \in$ Aut $U$. Suppose $\lambda$ is a limit ordinal and $\theta U_{\lambda}=U_{\lambda}$. By Lemma 3.1(b), letting $\alpha=\lambda$ and $\alpha=\lambda+1$ respectively, we have $\theta \tilde{F}_{i}^{\lambda}=\tilde{F}_{i}^{\lambda}$ and $\theta D_{i}^{\lambda+1}=D_{i}^{\lambda+1}$, and so by $3.7 \theta \tilde{I}_{i}^{\lambda}=\tilde{I}_{i}^{\lambda}$ for almost all $i \geqslant 0$. Now Lemma 3.4 (via $\sim$ ) implies $\theta_{\lambda} \in \operatorname{Inn} U_{\lambda}$ and, by Lemma 3.2, $\theta \in \operatorname{Inn} U$.

All that remains is to define the chain $\left\{F_{i}^{\lambda+1}\right\}$ in such a way that 3.7 holds.

We will use the notations of $1.5,1.8$, and 1.9 as follows. $C_{\lambda+1}=\cup H_{i}=$ $\cup F_{i}^{\lambda+1}, C_{\lambda}=\cup F_{i}^{\lambda}, f_{\lambda}\left(F_{i}^{\lambda}\right)=E_{i}<C_{\lambda+1}$, and $\mathrm{gp}\left(E_{i+1}, H_{i}\right)=J_{i}$. Note that

3.8. $J_{i}$ is generated by the amalgam

$$
A_{i}=\underbrace{E_{i+1}}_{E_{i}} H_{i}
$$

and is obtained (see 1.8) isomorphically from Lemma 2.1 (because of the construction of $\mathcal{Q}$ in 2.11), and

3.9. $f_{\lambda}\left(C_{\lambda}\right) \cap H_{i}=E_{i}$ (by 1.7 ).

The desired condition 3.7 is equivalent to

3.10. $f_{\lambda}\left(I_{i}^{\lambda}\right)=f_{\lambda}\left(C_{\lambda}\right) \cap F_{i}^{\lambda+1}$ (see 1.11).

There are two cases to be considered in defining $F_{i}^{\lambda+1}$.

Case I. $F_{i}^{\lambda}$ has the form $L_{n}$ or $L_{n} \oplus R_{n}^{j}, 1 \leqslant j \leqslant d-2$. In this case we define $F_{i}^{\lambda+1}=H_{i}$ and 3.10 is satisfied, using 3.9 , because $I_{i}^{\lambda}=F_{i}^{\lambda}$ by Definition 3.3.

Case II. $F_{i}^{\lambda}$ has the form $L_{n} \oplus A_{n}$. In this case $F_{i-1}^{\lambda}=L_{n}$ and $I_{i}^{\lambda}=L_{n} \oplus$ $B_{n}<L_{n} \oplus R_{n}^{1}=F_{i+1}^{\lambda} . F_{i}^{\lambda+1}$ must contain $H_{i-1}=F_{i-1}^{\lambda+1}$ (by Case I) and so, to satisfy 3.10 , it is natural to define

3.11. $F_{i}^{\lambda+1}$ is generated by the "subamalgam"

$$
A^{\prime}=f_{\lambda}\left(L_{n} \oplus B_{n}\right) \underbrace{H_{i-1}}_{E_{i-1}}
$$

of the amalgam $A_{i}$ of 3.8 . 
(For the definition of subamalgam see 3.13. That $A^{\prime}$ is a subamalgam of $A_{i}$ follows from 3.9 and the fact $f_{\lambda}\left(L_{n} \oplus B_{n}\right) \cap E_{i}=f_{\lambda}\left(L_{n} \oplus B_{n}\right) \cap f_{\lambda}\left(L_{n} \oplus A_{n}\right)$ $=f_{\lambda}\left(L_{n}\right)=E_{i-1}$.) Since $A_{i}<H_{i+1}$, we have

$$
F_{i}^{\lambda+1} \cap f_{\lambda}\left(C_{\lambda}\right)<H_{i+1} \cap f_{\lambda}\left(C_{\lambda}\right)=E_{i+1} \text { by } 3.9 \text {, }
$$

and so

$$
F_{i}^{\lambda+1} \cap f_{\lambda}\left(C_{\lambda}\right)=F_{i}^{\lambda+1} \cap E_{i+1} .
$$

Hence 3.10 will be satisfied just in case

3.12. $f_{\lambda}\left(L_{n} \oplus B_{n}\right)=F_{i}^{\lambda+1} \cap E_{i+1}$.

This condition will hold provided the group $J_{i}=\mathrm{gp}\left(A_{i}\right)$ of 3.8 has a certain "subamalgam property".

3.13. Definition of the SUbamalgam PROPERTY $S(A, G)$. If

$$
A=V_{E}
$$

is an amalgam, then $A_{0} \leqslant A$ is a subamalgam of $A$ if

$$
A_{0}={\stackrel{F}{F_{0}}}_{E_{0}}^{H_{0}}
$$

where $F_{0} \cap E=H_{0} \cap E=E_{0}, F_{0} \leqslant F$, and $H_{0} \leqslant H$.

The subamalgam property $S(A, G)$ holds if $G=\mathrm{gp}(A)$ and, for every subamalgam $A_{0}$ of $A$, we have $\operatorname{gp}\left(A_{0}\right) \cap A=A_{0}$.

So far we have observed

3.14. LEMMA If $S\left(A_{i}, J_{i}\right)$ holds for all $i$, where $A_{i}$ is the amalgam of 3.8, then $3.12 \Rightarrow 3.10 \Rightarrow 3.7$ all hold, and $U \in \mathcal{U}$ is a complete group by Lemma 3.6.

Proof. 3.12 follows directly from 3.11 and the property $S\left(A_{i}, J_{i}\right)$.

To insure that $S\left(A_{i}, J_{i}\right)$ holds it will be necessary, in view of 3.8, to obtain groups $G$ from Lemma 2.1 such that $S(A, G)$ holds where

$$
A=\stackrel{\varphi F}{V^{\prime}}
$$

The following definition will facilitate the proof of this: Suppose $G=\mathrm{gp}_{1}(A)$ and $Q=\mathrm{gp}_{2}(A)$ where

$$
A={ }_{E}^{F}
$$

The notation $\Psi: G \stackrel{A}{\rightarrow} Q$ will mean that $\Psi$ is a homomorphism and $\Psi(a)=a$ for all $a \in A$.

3.15. LemMa. Using the above notation, 
(a) Suppose $A_{0}$ is a subamalgam of $A, \mathrm{gp}_{2}\left(A_{0}\right) \cap A=A_{0}$, and $\Psi: G \stackrel{A}{\rightarrow} Q$. Then $\mathrm{gp}_{1}\left(A_{0}\right) \cap A=A_{0}$.

(b) Suppose $S(A, Q)$ holds and $\Psi: G \stackrel{A}{\rightarrow} Q$. Then $S(A, G)$ holds.

The proof is straightforward.

3.16. LEMMA. (a) Recall that in the proof of Lemma 2.1 we began with any group $Q=\mathrm{gp}_{1}(A)$,

$$
A=\stackrel{\varphi F}{V^{H}}
$$

and we then constructed a group $G=\mathrm{gp}_{2}(A)$ satisfying (i)-(iii). There is a map $\Psi: G \stackrel{A}{\rightarrow} Q$.

(b) Suppose $A$ is a finite amalgam and for each subamalgam $A_{0}$ of $A$ there is a finite group $G_{0}=\mathrm{gp}_{0}(A)$ such that $\mathrm{gp}_{0}\left(A_{0}\right) \cap A=A_{0}$. Then there is a finite group $G=\mathrm{gp}(A)$ such that $S(A, G)$ holds.

Proof of (a). Recall that $Q=\left(\varphi F{ }_{E} H\right) / K$ and $G=\left(\varphi F *{ }_{E} H\right) / N, N \leqslant$ $K$, where both $H$ and $\varphi F$ are identified with their images $\bmod K$ and $N$. If $\Psi$ is the factor map, then $\Psi: G \stackrel{A}{\rightarrow} Q$.

Proof of (b) Let

$$
A=\stackrel{F}{\bigvee^{H}}
$$

For each $A_{0}$ let $G_{0}=\left(F{ }^{*} H\right) / N_{0}$ and define $N=\cap\left\{N_{0}: A_{0}<A\right\}$ and $G=\left(F *_{E} H\right) / N$. $G$ is finite since there are only finitely many $A_{0}$. For each $A_{0}, \Psi_{0}: \stackrel{E_{A}}{\rightarrow} G_{0}$ where $\Psi_{0}$ is the factor map, and by 3.15 (a) we conclude $S(A, G)$ holds.

3.17. LeMMA. If for every finite amalgam

$$
A=\underbrace{}_{E} H
$$

there is a finite $Q=\mathrm{gp}(A)$ such that $S(A, Q)$ holds, then the groups $G$ of Lemma 2.1 can be obtained so that $S\left(A_{i}, J_{i}\right)$ holds for all $i$. With these choices for $G$ Lemma 3.14 implies that $U \in \mathcal{U}$ is a complete group.

Proof. To obtain the group $G$ of Lemma 2.1, we can begin with a group $Q$ such that $S(A, Q)$ holds. Then, by $3.16(\mathrm{a})$ and $3.15(\mathrm{~b}), S(A, G)$ also holds. Since $J_{i}=\mathrm{gp}\left(A_{i}\right)$ is obtained isomorphically from $G=\mathrm{gp}(A)$ (see 3.8 and 1.8), we have $S\left(A_{i}, J_{i}\right)$ also.

All that is left is to fulfil the hypothesis of Lemma 3.17. 
THEOREM. If

$$
A=\stackrel{F}{\searrow_{E}^{H}}
$$

is a finite amalgam, there is a finite group $G=\mathrm{gp}(A)$ such that $S(A, G)$ holds.

Proof. By Lemma 3.16 (b), we need to show that if

$$
A_{0}={\stackrel{F_{0}}{E_{0}}}^{H_{0}}
$$

there is a finite group $G=\operatorname{gp}(A)$ such that $\operatorname{gp}\left(A_{0}\right) \cap A=A_{0}$.

We will use the notation of [11, §3] with $A, B$ and $H$ replaced by $F, H$ and $E$ respectively, that is, $G$ will be constructed as a permutational product of $F$ and $H$ with a specific choice of left transversals, $S$ for $E$ in $F$, and $T$ for $E$ in $H$. We will choose $S$ and $T$ so that $S_{0} \leqslant S$ where $S_{0}$ is a transversal for $E_{0}$ in $F_{0}$ and $T_{0} \leqslant T$ where $T_{0}$ is a transversal for $E_{0}$ in $H_{0}$. Now $G=\operatorname{gp}(\rho F, \rho H)$ where each $\rho(a), a \in F$, and $\rho(b), b \in H$, is a permutation of the set $S \times T \times E$ induced by translation: $(s, t, e) \rho(a)=\left(s^{\prime}, t, e^{\prime}\right)$ where sea $=s^{\prime} e^{\prime}$ and $(s, t, e) \rho(b)=\left(s, t^{\prime}, e^{\prime}\right)$ where teb $=t^{\prime} e^{\prime}$. It follows that every permutation in $\operatorname{gp}\left(\rho F_{0}, \rho H_{0}\right)$ permutes the triples $S_{0} \times T_{0} \times E_{0}$ among themselves, but that no element of $\rho\left(F-F_{0}\right)$ or $\rho\left(H-H_{0}\right)$ has this property. Identifying $F$ with $\rho F$ and $H$ with $\rho H$, the theorem is proved.

Some Questions. Apart from the obvious question of extending these results to $\kappa>\kappa_{1}$, there is a question whether the present technique can be used to construct $U L F$ groups of order $\kappa_{1}$ satisfying more stringent conditions; and, somewhat closer to home, there are interesting problems concerning the structure of Hall's group $C$.

It would be interesting to see if the following questions can be approached by refining the choice of the amalgamating groups and the chains in this construction. Does there exist a ULF group of order $\aleph_{1}$ all of whose uncountable subgroups are (simple, complete, universal, etc.).

It was mentioned in the paragraph before 3.3 that some single chain $\left\{F_{i}^{\lambda}\right\}$ might suffice to smother the outer automorphisms of $C$. A possibility for such a chain is $\left\{D_{i}\right\}$ where $D_{i+1}$ is the symmetric group on $D_{i}$ and $D_{i}$ is embedded in $D_{i+1}$ as the diagonal of $R \oplus L$, the direct sum of the right and left regular representations of $D_{i}$ in $D_{i+1}$. It can be shown that the centralizer of $D_{i}$ in $D_{i+1}$ is 1 , and so the proof of Lemma 3.4 would show that any automorphism of $D=\cup D_{i}$ which stabilizes the chain $\left\{D_{i}\right\}$ is inner. Is $D \cong C$ ? If this could be shown, the last part of our proof would be unnecessary.

The automorphism group of $C$ might also merit some attention. We mentioned already that Aut $C$ contains a free group on $2^{\kappa_{0}}$ generators. Does 
Aut $C$ contain the symmetric group on a countable set? Is there an infinite set $S<C$ such that every permutation of $S$ can be lifted to an automorphism of $C$ ? Finally, we mention an interesting question of J. E. Roseblade. Does Inn $C=$ the locally finite radical of Aut $C$ ?

ADDED IN PROOF. Since this paper was written, S. Shelah has kindly shown us an elegant and compact "tree-limit" construction whereby $2^{\kappa_{0}}$ subgroupincomparable $U L F$ groups of power $2^{\mathrm{N}_{0}}$ are obtained. The property 0.2 is also met, and he conjectures that complete groups can also be obtained. His method also applies to e.c. groups and skew fields. The property 0.3 does not hold for the groups of Shelah's construction, but we could hardly expect it to, since, e.g., every e.c. group of power $\kappa$ possesses a subgroup of power $\kappa$ which contains a proper copy of itself, namely the centralizer of an element of infinite order.

\section{BIBLIOGRAPHY}

1. G. Baumslag, Lecture notes on nilpotent groups, Regional Conf. Ser. in Math., no. 2, Amer. Math. Soc., Providence, R. I., 1971. MR 44 \#315.

2. G. Fodor, On stationary sets and regressive functions, Acta Sci. Math. (Szeged) 27 (1960), 105-110. MR 34 \#66.

3. Philip Hall, Some constructions for locally finite groups, J. London Math. Soc. 34 (1959), 305-319.

4. K. Hickin, Countable type local theorems in algebra, J. Algebra 27 (1973), 523-537. MR 49 \#5179.

5. B. Jónsson, Homogeneous universal relational systems, Math. Scand. 8 (1960), 137-142. MR 23 \# A2328.

6. O. Kegel and B. Wehrfritz, Locally finite groups, North-Holland, Amsterdam, 1973.

7. A. Karrass and D. Solitar, The subgroups of a free product of two groups with an amalgamated subgroup, Trans. Amer. Math. Soc. 150 (1970), 227-255. MR 41 \#5499.

8. A. Macintyre and S. Shelah, Uncountable universal locally finite groups (to appear).

9. A. Macintyre, Existentially closed structures and Gentzen's principle (to appear).

10. M. Morley and R. L. Vaught, Homogeneous universal models, Math. Scand. 11 (1962), 37-57. MR 27 \#37.

11. B. H. Neumann, Permutational products of groups, J. Austral. Math. Soc. 1 (1959/60), 299-310. MR 23 \#A922.

12. _ An essay on free products of groups with amalgamations, Philos. Trans. Roy. Soc. London Ser. A. 246 (1954), 503-554. MR 16, 10.

13. W. R. Scott, Group theory, Prentice-Hall, Englewood Cliffs, N. J., 1964. MR 29 \#4785.

14. W. Sierpinski, Cardinal and ordinal numbers, 2nd rev. ed., Monografie Mat., vol., 34, PWN, Warsaw, 1965. MR 33 \#2549.

Department of Mathematics, Michigan State University, East lansing, Michigan 48824

Current address: Department of Mathematics, Yale University, New Haven, Connecticut 06520 